ARTICLE

Received 29 Mar 2014 | Accepted 27 May 2014 | Published 18 Jun $2014 \quad$ DOI: 10.1038/ncomms5218

\title{
Hidden one-dimensional spin modulation in a three-dimensional metal
}

\author{
Yejun Feng ${ }^{1,2}$, Jiyang Wang ${ }^{2}$, A. Palmer², J.A. Aguiar ${ }^{3}$, B. Mihaila 3,4 , J.-Q. Yan ${ }^{5,6}$, P.B. Littlewood ${ }^{2,7}$
}

\& T.F. Rosenbaum ${ }^{2}$

Pressure can transform a transparent material into an opaque one, quench the moments in a magnet and force solids to flow like liquids. At $15 \mathrm{GPa}$, the pressure found $500 \mathrm{~km}$ below the earth's surface, the semiconductors silicon and germanium superconduct. Yet, at this same pressure, we show here that the magnetism in metallic GdSi remains completely robust even as it shrinks by one-seventh of its volume. Non-resonant $X$-ray magnetic diffraction in a specially designed diamond anvil cell, combined with band structure calculations, reveal the stability of the incommensurate spin density wave, which can be traced to a persistently nested portion of the Fermi surface that becomes increasingly onedimensional under pressure. A cooperative interaction between nested, itinerant spins and local magnetic moments provides the organizing principle for the modulated magnetic order, salient both for its insights into the role of topology in ordered states and its potential functionality.

\footnotetext{
${ }^{1}$ The Advanced Photon Source, Argonne National Laboratory, Argonne, Illinois 60439, USA. ${ }^{2}$ The James Franck Institute and Department of Physics, University of Chicago, Chicago, Illinois 60637, USA. ${ }^{3}$ Materials Science and Technology Division, Los Alamos National Laboratory, Los Alamos, New Mexico 87545, USA. ${ }^{4}$ Physics Division, National Science Foundation, Arlington, Virginia 22230, USA. ${ }^{5}$ Department of Materials Science and Engineering, University of Tennessee, Knoxville, Tennessee 37996, USA. ${ }^{6}$ Materials Science and Technology Division, Oak Ridge National Laboratory, Oak Ridge, Tennessee 37831 , USA. ${ }^{7}$ Physical Sciences and Engineering, Argonne National Laboratory, Argonne, Illinois 60439, USA. Correspondence and requests for materials should be addressed to Y.F. (email: yejun@aps.anl.gov) or to T.F.R. (email: t-rosenbaum@uchicago.edu).
} 
O ne-dimensional materials display a variety of intricate collective states, from gapless Luttinger liquids with spin-charge separation to gapped, interleaved spin and charge density waves (SDWs and CDWs). Model onedimensional organic conductors such as (TMTTF) ${ }_{2} \mathrm{X}$ and TTF-TCNQ ${ }^{1-6}$ sit on the edge between spin and charge order and superconductivity. One-dimensional instabilities also exist within materials of higher dimension and can play a dominant role in determining their properties. For example, in the cubic A15 compound $\mathrm{V}_{3} \mathrm{~S}$, dense linear chains of $\mathrm{V}$ atoms provide an enhanced density of states at the Fermi surface, leading to an unexpectedly high superconducting transition temperature ${ }^{7}$. Similarly, Fermi surface nesting along particular directions in reciprocal space dominates the magnetic behaviour of threedimensional elemental $\mathrm{Cr}^{8}$ and the two-dimensional, rare-earth tritellurides 9 .

Recently, a new mechanism for SDW order was discovered in the rare-earth intermetallic GdSi at ambient pressure. The ordering wave vector is incommensurate with both the $\mathbf{b}^{*}$ - and $\mathbf{c}^{*}$-axes at $\mathbf{Q}=\left(0, q_{\mathrm{b}}, q_{\mathrm{c}}\right) \sim(0,0.483,0.092)$ due to a quasi-onedimensional Fermi surface instability ${ }^{10}$. The antiferromagnetic state is composed of nested itinerant electrons in a cooperative interaction with localized Gd $4 f$ moments: the itinerant electrons satisfying the nesting condition are coupled in a SDW via scattering from the local moments, while the local moments interact through the Ruderman-Kittel-Kasuya-Yosida indirect exchange mediated by the itinerant SDW and assume the ordering wave vector determined by the nesting. This selfconsistent, cooperative organization between local and itinerant spins is analogous to the interplay of lattice distortions and charge in phonon-mediated CDWs. Most SDWs and CDWs are highly susceptible to pressure ${ }^{11,12}$. However, the stability and evolution of the itinerant magnetic order as the separation between local moments is tuned in this new type of SDW has not been explored.
Hydrostatic pressure $P$ provides continuous fine-tuning capability as well as a vast range with $P>300 \mathrm{GPa}$ accessible in diamond anvil cells ${ }^{13}$. However, tracking magnetism under pressure is technically difficult and often limited to indirect or spectroscopic techniques, such as electrical transport ${ }^{6,14}$, magnetostriction, X-ray emission ${ }^{15}$, nuclear magnetic resonance $^{2-4}$ and Mossbauer. Neutron or X-ray magnetic diffraction needs to be employed for direct insight into the long-range magnetic order and the spin structure. The typical pressure range for neutron magnetic diffraction is $25 \mathrm{GPa}$ for powders $^{16}$ and $<10 \mathrm{GPa}$ for single crystals ${ }^{17}$. Single-crystal $\mathrm{X}$-ray magnetic diffraction has become competitive over the past decade as a direct probe of antiferromagnetism under pressure $^{18-20}$.

In the following sections, we present a combined experimental and theoretical approach from both non-resonant X-ray magnetic diffraction and $a b$ initio band structure calculations to elucidate the pressure evolution of the SDW ground state in GdSi. We use newly designed, wide-angle, partially perforated diamond anvils to increase signal throughput and reduce background (Methods; Fig. 1). This improvement in high-pressure techniques has allowed us to track directly the SDW ground state in GdSi with quantitative measurements of both the magnetic order parameter and the wave vector for pressures up to $17 \mathrm{GPa}$. Both quantities remain stable even as the lattice varies significantly, until a firstorder structural transition from monoclinic to orthorhombic occurs. Using band structure calculations, the stability of the antiferromagnetism can be traced back to a persistently nested Fermi surface, which grows more and more one-dimensional under pressure during a charge transfer process between different itinerant bands.

\section{Results}

High-resolution X-ray diffraction. We show in Fig. 1 representative scans of both the structural and magnetic order at high
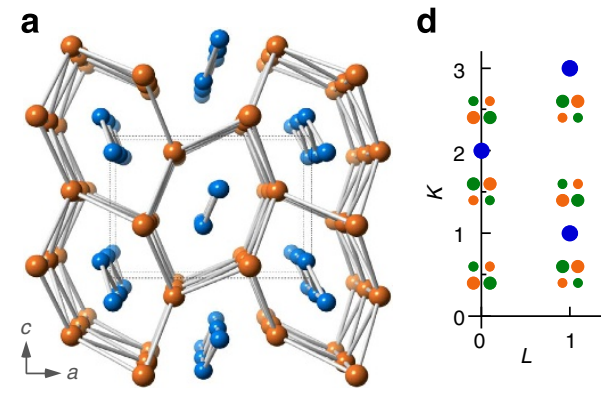

b
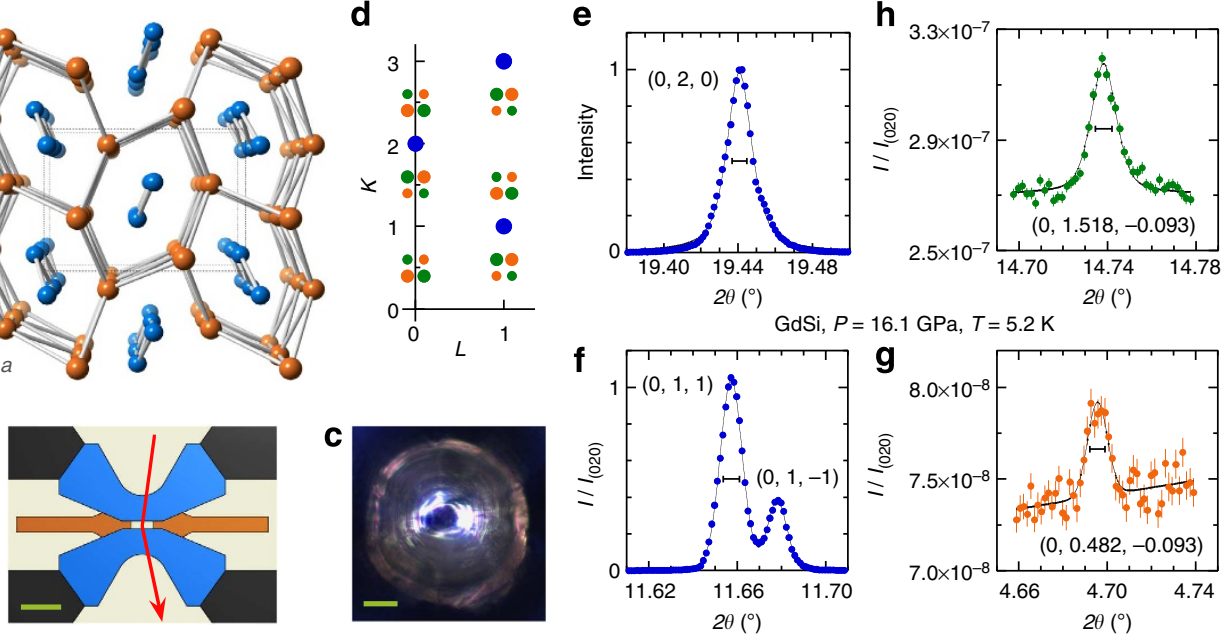

Figure 1 | High-pressure X-ray magnetic diffraction. (a) Lattice structure of GdSi, viewed along the $b$ axis, with a depth of three unit cells. All bonds between $\mathrm{Gd}$ (orange) and $\mathrm{Si}$ (blue) are removed to highlight the linear nature of double Si chains surrounded by Gd tubes. (b) A schematic of a new type of perforated diamond anvil designed for $X$-ray magnetic diffraction. X-rays (red lines with arrow) diffract off the sample (not shown) from inside the pressure chamber, which is constructed with two Boehler-Almax type of diamond anvils with wide-angle perforation (blue) and a gasket (orange). Scale bar, $0.8 \mathrm{~mm}$. (c) Sample chamber assembly viewed through one of the perforated diamond anvils. Both sample (rectangular shadow in the middle) and silver manometer (triangular shadow) can be seen in the 400- $\mu$ m diameter sample chamber. Scale bar, $0.1 \mathrm{~mm}$. (d) Schematic of the diffraction patterns of both lattice (blue) and incommensurate magnetic order (orange and green) of GdSi in the $K-L$ plane. Orange and green colours correspond to two degenerate magnetic domains. Magnetic diffraction intensities surrounding the forbidden lattice orders such as $(0,1,0)$ are much weaker. (e-h) Longitudinal scans of both lattice and magnetic order. Each peak is fit with a pseudo-Voigt function, in addition to an extra linear background for magnetic peaks (g,h). Vertical error bars represent $1 \sigma$ s.d. of counting statistics. The horizontal bars at the middle of each peak represent the FWHM of the instrument resolution. All intensities are normalized to that of the $(0,2,0)$ Bragg peak. 
a
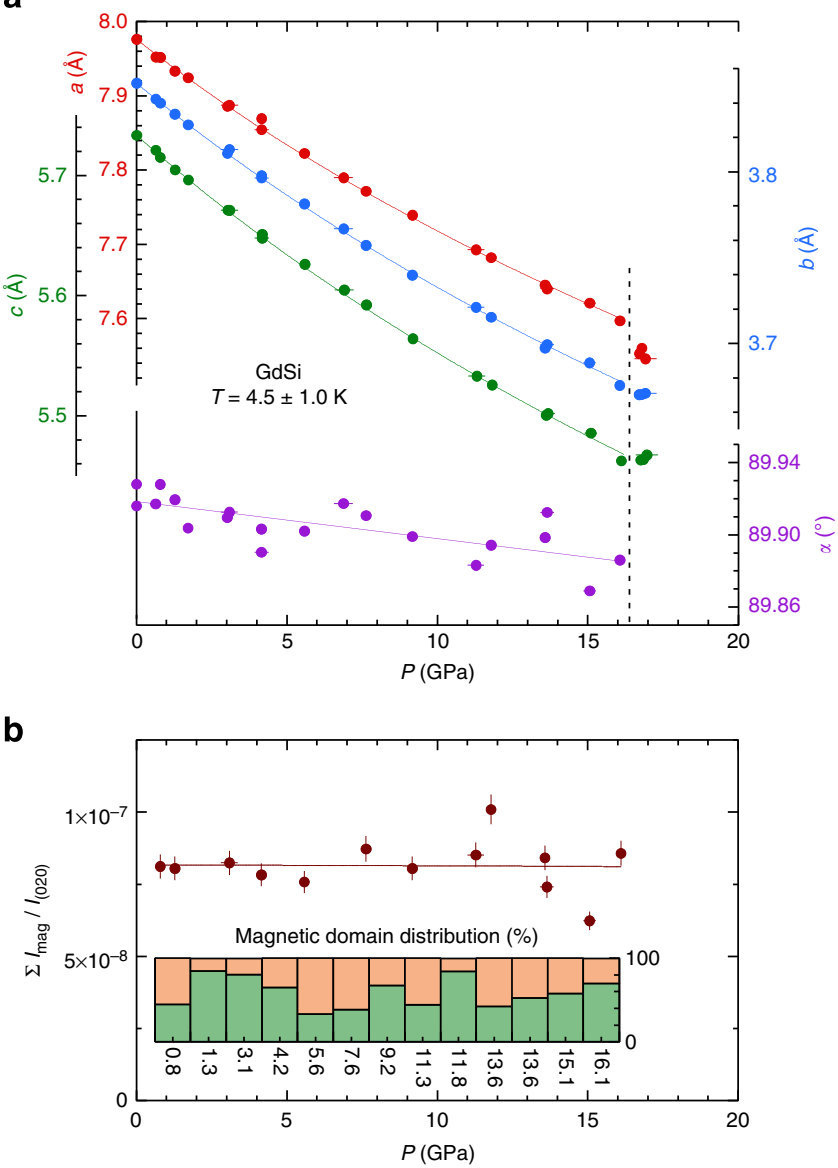

Figure 2 | Pressure dependence of both lattice structure and magnetic order parameter. (a) Lattice constants, $a, b, c$, and the monoclinic angle $\alpha$ as a function of pressure. Below $P_{\mathrm{c}}=16.4 \mathrm{GPa}$, lattice constants $a, b, c$ are fit with the single-parameter Birch equation of state specified in the text. $\alpha(P)$ is fit linearly. Above $P_{c}$, there is a first-order structural transition to an orthorhombic phase. (b) The magnetic order parameter $I_{\text {mag }} / I_{(020)}$ is summed over diffraction intensities at $\left(0,2-q_{\mathrm{b}}, \pm q_{\mathrm{c}}\right)$ and $\left(0, q_{\mathrm{b}}, \pm q_{\mathrm{c}}\right)$ for both magnetic domains. (Inset) Magnetic domain distribution at each pressure point where the order parameter was measured. Error bars represent $1 \sigma$ s.d.

pressure and low temperature, $P=16.1 \mathrm{GPa}$ and $T=5.2 \mathrm{~K}$, exemplifying the high-resolution X-ray magnetic diffraction technique. GdSi has a collinear spin structure between the Néel temperature, $T_{\mathrm{N}}=54.5 \mathrm{~K}$, and the spin-flip transition temperature, $T_{\mathrm{SF}}=53.0 \mathrm{~K}$, and an orthorhombic lattice structure. Below $T_{\mathrm{SF}}$ at ambient pressure, the spin structure is planar and is associated with a monoclinic lattice structure (space group $\mathrm{P} 2{ }_{1} / \mathrm{c}$ ) that can be detected by a split of the $d$-spacing between $(0, K, L)$ and $(0, K,-L)^{10}$. There are then two magnetic domains with $\mathbf{Q}$ vectors along $\left(0, \pm q_{\mathrm{b}}, q_{\mathrm{c}}\right)$ associated with the individual monoclinic lattice domains (see Fig. 1d and Fig. 2b, inset). At $16.1 \mathrm{GPa}$, the highest pressure that antiferromagnetism was directly observed, the monoclinic splitting of the $(0,1,1)$ order remains clear (Fig. 1f), although the longitudinal line shapes are slightly broadened above the instrument resolution ${ }^{21}$. Here, both the spontaneously twinned single crystals and near-resolutionlimited diffraction profiles testify to the quasi-hydrostatic pressure condition in the sample environment. At $16.1 \mathrm{GPa}$, the full widths at half maximum (FWHMs) of the peaks give a coherence length of $1,400 \AA$ for both the lattice and the antiferromagnetic order.

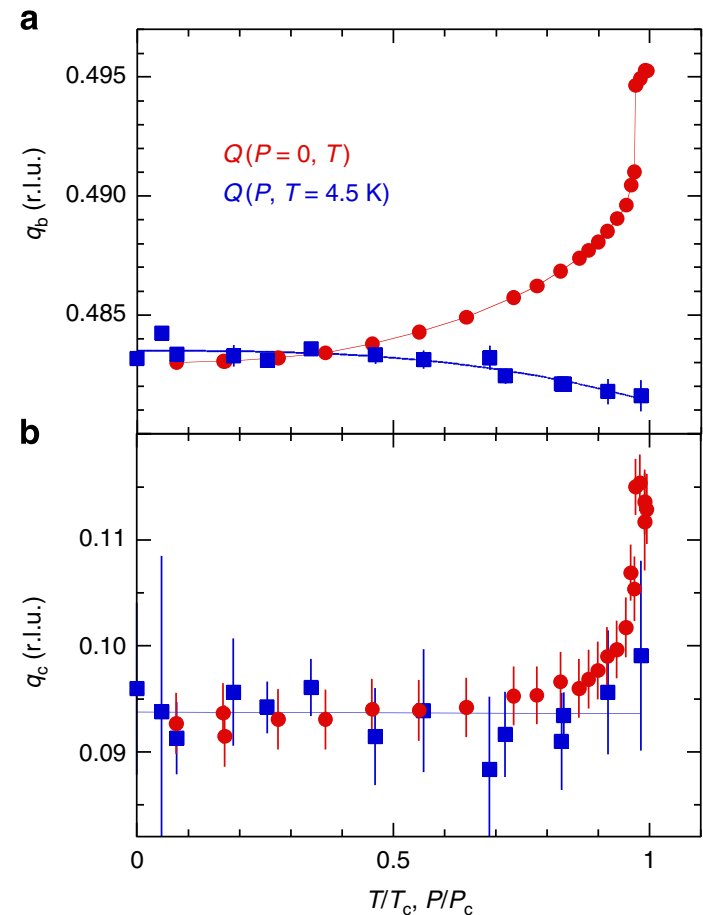

Figure 3 | Temperature and pressure dependence of the magnetic wave vector. $(\mathbf{a}, \mathbf{b})$ Evolution of the incommensurate wave vector $\mathbf{Q}=\left(0, q_{b}, q_{c}\right)$ in reciprocal lattice unit (r.l.u.) towards the pressure- and temperatureinduced phase boundaries. The Fermi surface nesting condition changes little with $P$ at low $T$. Horizontal axes are normalized by $P_{\mathrm{c}}$ and $T_{\mathrm{N}}$, respectively. Error bars represent $1 \sigma$ s.d. Solid lines are guides to the eye. The discontinuity in $\mathbf{Q}(T)$ happens at the spin-flip transition $T_{\text {SF }}$.

We plot in Fig. 2a the substantial change in the lattice constants with $P$. Each refined lattice constant, $a, b$ and $c$, is fit with the single-parameter Birch equation of state: $P=3 / 2$ $B_{l}\left[\left(l_{0} / l\right)^{7}-\left(l_{0} / l\right)^{5}\right]$, where $l_{0}$ is the individual lattice constant at ambient pressure. The three bulk moduli, $B_{\mathrm{a}}=83.1 \pm 0.4 \mathrm{GPa}$, $B_{\mathrm{b}}=88.0 \pm 0.3 \mathrm{GPa}$ and $B_{\mathrm{c}}=85.4 \pm 0.4 \mathrm{GPa}$, are close in value, indicating that the crystal structure evolves in a nearly isotropic fashion under pressure. Overall, the antiferromagnetism in GdSi persists over a $4.7 \%$ compression of the linear lattice constants and a $13 \%$ reduction in volume. At ambient pressure, the monoclinic angle $\alpha$ of the lattice tracks the evolution of the planar magnetic order via magnetostrictive coupling ${ }^{10}$. Up to $16.1 \mathrm{GPa}$, $\alpha$ changes only slightly and deviates slowly away from $90^{\circ}$. At a critical pressure, $P_{\mathrm{c}}=16.4 \mathrm{GPa}$, the a-axis lattice constant shows a clear discontinuity of $\Delta a / a=5.9 \times 10^{-3}$, while changes along the other two axes are minimal. Beyond $P_{\mathrm{c}}$, no splitting of the $(0, K$, $L$ ) orders is observed for three different crystals as the lattice structure again becomes orthorhombic (the structure found for $T>T_{\mathrm{SF}}$ at ambient pressure). The incommensurate antiferromagnetic order is no longer visible beyond $P_{c}$, and the nature of the high-pressure phase, whether it is a paramagnet, a ferromagnet or a commensurate antiferromagnet, cannot be discerned from this data.

Antiferromagnetic ground state under pressure. The incommensurate antiferromagnetism in GdSi and its evolution with $P$ can be probed directly by observing the magnetic order at points in reciprocal space such as $\left(0, q_{\mathrm{b}}, \pm q_{\mathrm{c}}\right),\left(0,2-q_{\mathrm{b}}, \pm q_{\mathrm{c}}\right)$ and $\left(0,2+q_{\mathrm{b}}, \pm q_{\mathrm{c}}\right)$. The magnetic nature of these incommensurate diffraction peaks was demonstrated previously at ambient pressure via resonant X-ray diffraction, and no lattice (charge) 


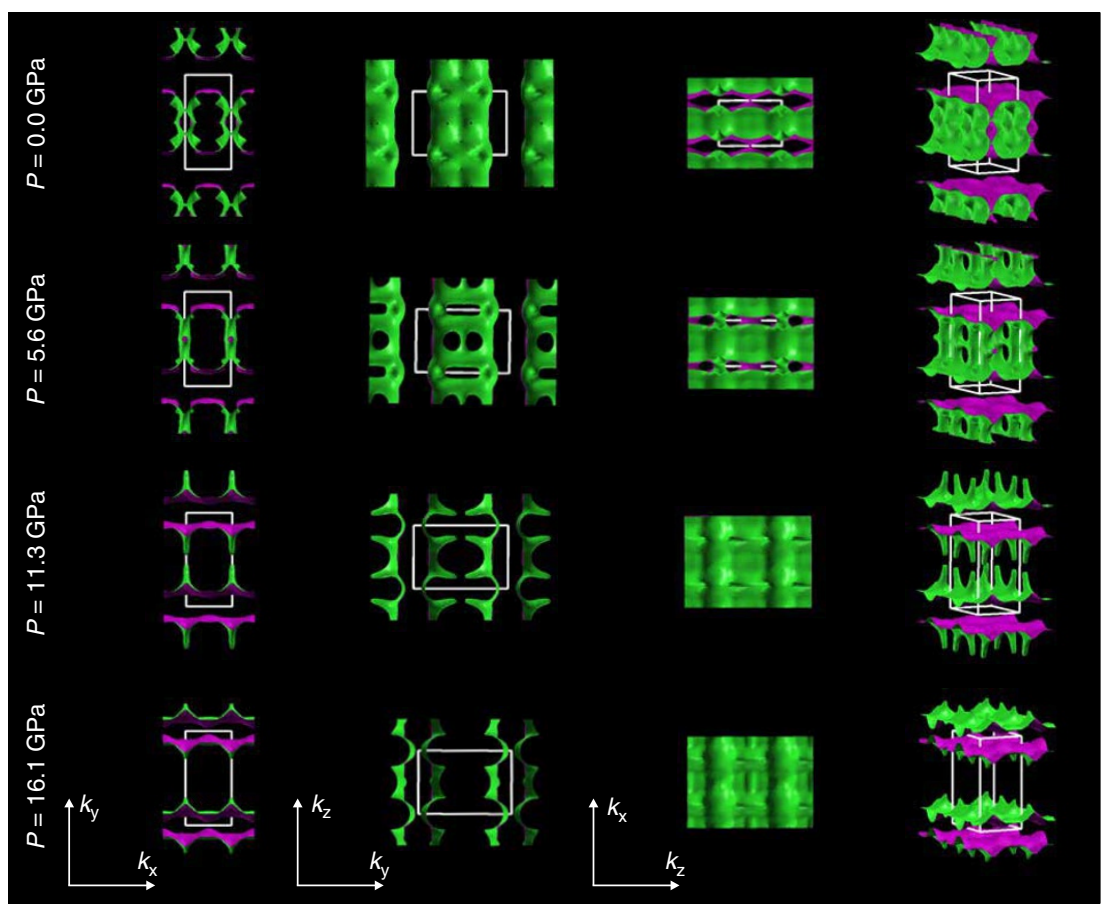

Figure 4 | Evolution of the nesting band under pressure. The calculated major itinerant band at the Fermi surface projected along each of three major reciprocal lattices, together with a three-dimensional perspective. The band structure calculations were carried out at four different pressures using the structural data of Fig. 2a. As pressure increases, a charge transfer process slowly depletes the band, but the nesting condition remains stable and grows progressively more one-dimensional.

coupling at this wave vector was detected ${ }^{10}$. As shown in Fig. $2 \mathrm{~b}$, and in telling contrast to the behaviour of the lattice, the magnetic order parameter remains unchanged over the entire range of pressures, $0 \leq P<P_{c}=16.4 \mathrm{GPa}$. The magnetic order parameter was measured by summing over the diffraction intensities of both domains (Fig. 2b, inset). In the diffraction geometry of a diamond anvil cell, it is difficult to measure a full azimuthal dependence under pressure ${ }^{10}$. However, given that the data were taken at a fixed azimuthal angle (see Methods), both the constant diffraction intensity and lattice monoclinic angle substantiate the interpretation that the spin structure is invariant under pressure.

In addition to the strength of the magnetic order parameter, the stability of the incommensurate spin structure in GdSi under pressure is further illustrated through measurements of the wave vector $\mathbf{Q}$. We show in Fig. 3, $\mathbf{Q}(P)$ at fixed $T$ and $\mathbf{Q}(T)$ at fixed $P$ at the approaches to the quantum and thermal transitions, respectively. Along the temperature path, there is a significant change in $\mathbf{Q}(T)$ that accelerates near the phase boundary in a manner characteristic of many incommensurate SDW and CDW systems $^{8,22}$. Under pressure, however, $\mathbf{Q}(P)$ of $\mathrm{GdSi}$ stays essentially constant (relative to the lattice) as it approaches the first-order, high-pressure phase boundary, with a slight, monotonic decrease in $q_{\mathrm{b}}(P)$; the germane characteristics of the Fermi surface nesting are unaffected. We note that $\mathbf{Q}(P)$ would behave differently for systems approaching a continuous quantum phase transition. A non-monotonic $\mathbf{Q}\left(P \rightarrow P_{c}\right)$ has been measured in the incommensurate SDW in $\mathrm{Cr}$ arising from fluctuation effects at the approach to the quantum critical point ${ }^{11}$. Here, in $\mathrm{GdSi}$, the magnetism does not weaken with applied pressure and quantum fluctuations have been cut off by the first-order structural transition.

\section{Discussion}

Band structure calculations provide insight into the unusual hardiness of the incommensurate SDW in GdSi. At ambient pressure, the paramagnetic band structure of GdSi possesses four different itinerant bands at the Fermi surface. The band with the nested features takes up the majority of the density of states; the other three bands present as either small pockets or tubes. We plot in Fig. 4 projections and a three-dimensional perspective of the nested major band at four different pressures from 0 to 16.1 GPa (calculations for all the bands can be found in the Supplementary Fig. 1). This dominant band persists throughout the entire pressure range. The nesting condition is reinforced as the band gradually assumes a sheet-like shape at $16.1 \mathrm{GPa}$ with a one-dimensional dispersion. A charge transfer effect slowly depletes its electronic density of states to the other minor bands. In addition, two extra bands start to emerge at the Fermi surface for pressures above $11 \mathrm{GPa}$, due to either charge transfer or bandwidth broadening (Supplementary Fig. 1). However, the quasi-one-dimensional nesting vector-which would dominate the wave vector selection of the SDW-is essentially unchanged with pressure.

Line-shape analysis of the resonant X-ray magnetic diffraction in GdSi at ambient pressure had illustrated the quasi-onedimensional characteristics of the nested Fermi surface ${ }^{10}$. Between the two components of itinerant and local spins that collectively form the SDW, the localized Gd $4 f$ moments lie $\sim 8 \mathrm{eV}$ below the Fermi level ${ }^{23}$. In pure $\mathrm{Gd}$, those $4 f$ spins would remain localized up to $106 \mathrm{GPa}^{15}$. The stability of the antiferromagnetism in $\mathrm{GdSi}$ with $P$ up to $16.4 \mathrm{GPa}$, as demonstrated by both its order parameter and its $\mathbf{Q}$-vector, is then clearly connected to the stability of the nested itinerant band, which progressively becomes one-dimensional under pressure. Above that pressure, some mix of charge transfer and a first-order structural transition destroys the major nested band and cuts off the incommensurate SDW order in GdSi.

The stability of antiferromagnetic order with large variations in the lattice spacing is unusual and raises fundamental physics and technological questions ${ }^{20}$. Very few examples exist in the 
literature for pressures above $10 \mathrm{GPa}$. High-pressure neutron powder diffraction on cubic $\mathrm{Gd} X(X=\mathrm{Bi}$, Sb, As) compounds shows that antiferromagnetic order can survive to at least 17, 19.5 and $43 \mathrm{GPa}$, respectively ${ }^{16}$. These are commensurate orders of the $(1 / 2,1 / 2,1 / 2)$ type that are locked into the lattice. For the incommensurate spin structure in $\mathrm{GdSi}$, the stability in the hidden one-dimensional Fermi surface can be traced, at least in part, to particular features in the material's structure. Although the magnetic order wave vector $\mathbf{Q}$ is incommensurate to both the $\mathbf{b}^{\star}$ and $\mathbf{c}^{\star}$ axes, it lies close to the $\mathbf{b}^{\star}$ direction. Sighting along the b axis (Fig. 1a), one sees Si atoms forming double chains inside a tubular Gd framework with a distorted hexagonal shape. This linear structural feature in real space is likely to be the source of the one-dimensional characteristics of the Fermi surface, as the band structure calculations reveal a strong character of Si carriers in the major nesting band.

It is also instructive to compare the hidden one-dimensional SDW order in GdSi with correlated electron states in quasi-onedimensional organic conductors such as TTF-TCNQ and $(\mathrm{TMTTF})_{2} \mathrm{X}$. The transition temperatures of all CDW, SpinPeierls and antiferromagnetic/SDW states are generally found to vary slowly with pressure tuning ${ }^{2,4,6}$, except in narrow crossover regions between two different ground states ${ }^{3,14}$. Direct probes of the order parameter and wave vector evolution with $P$ in these materials, presently very limited ${ }^{24}$, would be useful to explore the underlying characteristics of stability. More generally, organization principles that are robust against external disturbances-a generalized invar effect-provide opportunities for applications, ranging from giant magnetoresistance, multilayer devices ${ }^{25}$ to non-Abelian states in potential quantum computation application ${ }^{26}$. In $\mathrm{GdSi}$, the cooperative interaction between itinerant and local spin moments provides a robust magnetic ordering mechanism over a large strain range, which matches well with the typical range of elastic deformation for most functional materials ${ }^{27}$. Potentially, GdSi could be grown in thin film form, where the antiferromagnetism is expected to persist for significant lattice mismatches, and it could be a robust interfacial component in spin-valve and giant magnetoresistance devices ${ }^{25}$. The newly developed technical capability to examine directly incommensurate magnetism under tens of GPa pressure using X-ray magnetic diffraction provides a new means to continuously trace the interplay between spin, charge, itinerant and local degrees of freedom in functional materials.

\footnotetext{
Methods

X-ray diffraction. Non-resonant X-ray magnetic diffraction under pressure was performed at beamline 4-ID-D of the Advanced Photon Source ${ }^{19,20}$. A focused $20,000 \mathrm{keV} X$-ray beam was reduced to $100 \times 100 \mu^{2}$ size by motorized slits to match typical sample sizes around $80 \times 80 \times 30 \mu \mathrm{m}^{3}$ under pressure. Miniature single-crystal GdSi samples were prepared in successive stages by cutting with electron discharge machining, polishing to final thickness and dicing with an infrared laser to final lateral dimensions. Additional specifications on sample growth can be found in ref. 10. A NaI detector was used for measuring X-ray intensity, but no polarization analyser was used for magnetically diffracted X-rays. Using a six-circle Huber $\psi$-diffractometer in the vertical diffraction geometry, a high resolution in momentum space better than $1 \times 10^{-3} \AA^{-1}$ FWHM is achieved for longitudinal scans ${ }^{11,20,21}$. All measurements were made at the base temperature of the cryostat, which varied between 3.5 and $5.5 \mathrm{~K}$ for various cooldowns. A total of seven samples were studied under pressure with three of them compressed across the phase transition. Complete data sets of both magnetic order parameter and wave vector were collected on four samples, although magnetic diffraction was observed on all six samples probed below $P_{\mathrm{c}}$. The magnetic order parameter was measured at a constant azimuthal angle $\varphi=42^{\circ} \pm 2^{\circ}$, where $\varphi=0$ is for the azimuthal vector $(0,0,1)$ inside the diffraction plane. This geometry provides sensitivity to spins aligned along both the $a$ and $c$ axes in the planar spin structure ${ }^{10}$. The $\mathbf{Q}$ vector is calculated through measured $\left(0,2+q_{\mathrm{b}}, \pm q_{\mathrm{c}}\right)$ $\left(0,2-q_{\mathrm{b}}, \pm q_{\mathrm{c}}\right),\left(0, q_{\mathrm{b}}, \pm q_{\mathrm{c}}\right)$ and $(0,2,0)$ orders, normalized to the lattice constants $b$ and $c$ at each $P$.
}

High-pressure environment. Pressure cells with conical Boehler-Almax type of diamond anvils $\left(70^{\circ}\right.$ total $2 \theta$ angle) were used for single-crystal diffraction. We specifically designed a wide-angle $\left(2 \theta=60^{\circ}\right)$ partial perforation for all our diamond anvils (Fig. 1a). The partial perforation ends in a spherically curved shape ( $500 \mu \mathrm{m}$ radius) to better distribute stress in the anvils under compression. A pair of these partially perforated anvils leaves 0.9 to $1.0 \mathrm{~mm}$ total diamond thickness in the X-ray path. By comparison with the typical total diamond thickness of $3.2-4.5 \mathrm{~mm}$ with normal anvils, this wide-angle partial perforation design significantly reduces the elastic X-ray scattering background, while still reaching at least $17 \mathrm{GPa}$ for $800 \mu \mathrm{m}$ culet-sized anvils. The pressure medium was a $4: 1$ methanol:ethanol solution, and the cells were equipped with a helium-membrane controlled in situ pressure tuning mechanism at low temperature ${ }^{21}$. At $16.1 \mathrm{GPa}$, the pressure inhomogeneity across our sample is estimated to be either $0.30 \mathrm{GPa}$ FWHM using a comparison of the FWHM of lattice diffraction with that of the instrument resolution (Fig. 1), accounting for the sample-specific bulk moduli ${ }^{28}$, or a full range of $\pm 0.19 \mathrm{GPa}$ from previous pressure chamber studies under similar conditions ${ }^{21}$. The pressure inhomogeneity is typically linearly proportional to the absolute pressure in our assembly ${ }^{21}$.

Band structure calculations. First-principle band structure calculations were performed in the WIEN2K implementation of the generalized gradientapproximation approach ${ }^{29}$ using the full-potential linearized-augmented-planewave method ${ }^{30}$ with local orbitals for a better variational flexibility in the radial basis functions ${ }^{31}$. For non-magnetic calculations, spin-orbit effects were taken into account using a second-order variational step using scalar-relativistic eigenfunctions as basis ${ }^{32}$. A modified tetragonal method ${ }^{33}$ (with a k-mesh of $7 \times 14 \times 9$ ) was used for the k-point integration in Brillouin zone. Plots of the Fermi surface were generated using XCRYSDEN ${ }^{34}$ with code available from http:// www.xcrysden.org/. We also assumed all relative atomic positions are unchanged under pressure, and treated the lattice in the orthorhombic basis given the symmetry in the paramagnetic phase ${ }^{10}$

\section{References}

1. Voit, J. One-dimensional Fermi liquids. Rep. Prog. Phys. 57, 977-1116 (1994),

2. Klemme, B. J. et al. Commensurate and incommensurate spin-density wave and a modified phase diagram of the Bechgaard salts. Phys. Rev. Lett. 75, 2408-2411 (1995).

3. Chow, D. S. et al. Singular behavior in the pressure-tuned competition between spin-Peierls and antiferromagnetic ground states of (TMTTF $)_{2} \mathrm{PF}_{6}$. Phys. Rev. Lett. 81, 3984-3987 (1998).

4. Yu, W. et al. Electron-lattice coupling and broken symmetries of the molecular salt (TMTTF) ${ }_{2} \mathrm{SbF}_{6}$. Phys. Rev. B 70, 121101 (2004).

5. Foury-Leylekian, P. et al. Neutron-scattering evidence for a spin-Peierls ground state in (TMTTF) ${ }_{2} \mathrm{PF}_{6}$. Phys. Rev. B 70, 180405 (2004)

6. Yasuzuka, S., Murata, K., Arimoto, T. \& Kato, R. Temperature-pressure phase diagram in TTF-TCNQ: strong suppression of charge-density-wave state under extremely high pressure. J. Phys. Soc. Jpn 76, 033701 (2007).

7. Testardi, L. R. Structural instability and superconductivity in A15 compounds Rev. Mod. Phys. 47, 637-648 (1975).

8. Fawcett, E. Spin-density-wave antiferromagnetism in chromium. Rev. Mod. Phys. 60, 209-283 (1988).

9. Moore, R. G. et al. Fermi surface evolution across multiple charge density wave transitions in $\mathrm{ErTe}_{3}$. Phys. Rev. B 81, 073102 (2010).

10. Feng, Y. et al. Incommensurate antiferromagnetism in a pure spin system via cooperative organization of local and itinerant moments. Proc. Natl Acad. Sci. USA 110, 3287-3292 (2013).

11. Jaramillo, R. et al. Breakdown of the Bardeen-Cooper-Schrieffer ground state at a quantum phase transition. Nature 459, 405-409 (2009).

12. Berthier, C., Molinié, P. \& Jérome, D. Evidence for a connection between charge density waves and the pressure enhancement of superconductivity in 2H-NbSe ${ }_{2}$. Solid State Commun. 18, 1393-1395 (1976).

13. Akahama, Y. \& Kawamura, H. Pressure calibration of diamond anvil Raman gauge to 310GPa. J. Appl. Phys. 100, 043516 (2006).

14. Doiron-Leyraud, N. et al. Correlation between linear resistivity and $T_{c}$ in the Bechgaard salts and the pnictide superconductor $\mathrm{Ba}\left(\mathrm{Fe}_{1-\mathrm{x}} \mathrm{Co}_{\mathrm{x}}\right)_{2} \mathrm{As}_{2}$. Phys. Rev B 80, 214531 (2009).

15. Maddox, B. R. et al. $4 f$ delocalization in Gd: inelastic X-ray scattering at ultrahigh pressure. Phys. Rev. Lett. 96, 215701 (2006).

16. Goncharenko, I. N., Mirebeau, I. \& Ochiai, A. Magnetic neutron diffraction under pressures up to $43 \mathrm{GPa}$. Study of the $\mathrm{EuX}$ and $\mathrm{GdX}$ compounds. Hyperfine Interact. 128, 225-244 (2000).

17. Mignot, J.-M., Goncharenko, I. N., Link, P., Matsumura, T. \& Suzuki, T. Single-crystal neutron diffraction under high pressures: valence instabilities in Tm monochalcogenides. Hyperfine Interact. 128, 207-224 (2000).

18. Braithwaite, D. et al. High-pressure resonant magnetic X-ray diffraction and transport experiments in $\mathrm{Ce}\left(\mathrm{Fe}_{1-\mathrm{x}} \mathrm{Co}_{\mathrm{x}}\right)_{2}$. Phys. B 378-380, 782-783 (2006).

19. Feng, Y. et al. Pressure-tuned spin and charge ordering in an itinerant antiferromagnet. Phys. Rev. Lett. 99, 137201 (2007). 
20. Wang, J. et al. Pressure tuning of competing magnetic interactions in intermetallic $\mathrm{CeFe}_{2}$. Phys. Rev. B 86, 014422 (2012).

21. Feng, Y., Jaramillo, R., Wang, J., Ren, Y. \& Rosenbaum, T. F. High-pressure techniques for condensed matter physics at low temperature. Rev. Sci. Instrum. 81, 041301 (2010)

22. Moudden, A. H., Axe, J. D., Monceau, P. \& Levy, F. $q_{1}$ charge-density wave in $\mathrm{NbSe}_{3}$. Phys. Rev. Lett. 65, 223-226 (1990).

23. Abdelouahed, S., Baadji, N. \& Alouani, M. Electronic structure and x-ray magnetic circular dichroism of gadolinium beyond the local spin density approximation. Phys. Rev. B 75, 094428 (2007).

24. Megtert, S., Comes, R., Vettier, C., Pynn, R. \& Garito, A. F. Structural evidence of $2 \mathrm{k}_{\mathrm{F}}$ commensurability effects in TTF-TCNQ under high pressure. Solid State Commun. 37, 875-877 (1981).

25. Wolf, S. A. et al. Spintronics: a spin-based electronics vision for the future. Science 294, 1488-1495 (2001).

26. Stern, A. Non-Abelian states of matter. Nature 464, 187-193 (2010).

27. Zhou, M. Exceptional properties by design. Science 339, 1161-1162 (2013).

28. Jaramillo, R. et al. Chromium at high pressures: weak coupling and strong fluctuations in an itinerant antiferromagnet. Phys. Rev. B 77, 184418 (2008).

29. Perdew, J. P., Burke, K. \& Ernzerhof, M. Generalized gradient approximation made simple. Phys. Rev. Lett. 77, 3865-3868 (1996).

30. Blaha, P., Schwarz, K., Madsen, G. K. H., Kvasnicka, D. \& Luitz, J. WIEN2K, An Augmented Plane Wave Plus Local Orbitals Program for Calculating Crystal Properties (Technische Universität Wien, 2001).

31. Kunes, J., Novák, P., Schmid, R., Blaha, P. \& Schwarz, K. Electronic structure of fcc Th: Spin-orbit calculation with $6 p_{1 / 2}$ local orbital extension. Phys. Rev. B 64, 153102 (2001)

32. MacDonald, A. H., Pickett, W. E. \& Koelling, D. D. A linearised relativistic augmented-plane-wave method utilising approximate pure spin basis functions. J. Phys. C 13, 2675-2683 (1980).

33. Fradkin, M. A. External field in the Landau theory of a weakly discontinuous phase transition: Pressure effect in the martensitic transitions. Phys. Rev. B 50, 16326-16339 (1994)
34. Kokalj, A. Computer graphics and graphical user interfaces as tools in simulations of matter at the atomic scale. Comput. Mater. Sci. 28, 155-168 (2003).

\section{Acknowledgements}

The work at the University of Chicago was supported by the National Science Foun dation (NSF) grant number DMR-1206519. The work at the Advanced Photon Source of Argonne National Laboratory was supported by the U.S. Department of Energy Basic Energy Sciences (DOE-BES) under contract number NEAC02-06CH11357. Both P.B.L. and work at ORNL were supported by the Materials Sciences and Engineering Division, U.S. DOE-BES. Work at LANL was supported in part by the U.S. DOE. B.M. acknowledges support from NSF through its employee IR/D programme. A.P. was supported in part by DOE-SCGF under contract number DE-AC05-06OR23100.

\section{Author contributions}

Y.F. and T.F.R. designed the research. J.-Q.Y. grew and characterized samples. Y.F., J.W. and A.P. performed the measurement. B.M., J.A.A. and P.B.L. provided theoretical input and calculation. Y.F. and T.F.R. analysed the data and prepared the manuscript. All authors commented on the manuscript.

\section{Additional information}

Supplementary Information accompanies this paper at http://www.nature.com/ naturecommunications

Competing financial interests: The authors declare no competing financial interests.

Reprints and permissions information is available online at http://npg.nature.com/ reprintsandpermissions/

How to cite this article: Feng, Y. et al. Hidden one-dimensional spin modulation in a three-dimensional metal. Nat. Commun. 5:4218 doi: 10.1038/5218 (2014). 